\title{
THE IMPORTANCE OF VIRAL ETIOLOGY DETERMINATION IN SEVERE LOWER RESPIRATORY TRACT INFECTIONS IN INFANTS AND CHILDREN
}

\author{
Alexandru-Ioan Ulmeanu ${ }^{1,2}$, Carmen Zapucioiu ${ }^{1,2}$, Maria Dorina Craciun ${ }^{1}$, \\ Coriolan Ulmeanu ${ }^{1,2}$ \\ 1 "Grigore Alexandrescu" Emergency Children's Hospital, Bucharest \\ ${ }^{2}$ Pediatrics Department, "Carol Davila” University of Medicine and Pharmacy, \\ Bucharest
}

\begin{abstract}
Introduction. Community-acquired pneumonia is one of the leading causes of death worldwide in children under 5 years. In practice, viral detection using multiplex PCR is becoming increasingly used, it is most useful to understand the epidemiology and impact of viruses on the pediatric population but there are still many debates regarding the influence of viral detection on patient care.

Objectives. In this study we aimed to analyze the impact of viral etiology determination on the duration of hospitalization and on the administered treatment.

Material and methods. The selection criteria was: children between 0-5 years with severe lower respiratory tract infection. We have analyzed three groups of patients: a group with specified viral infection, a second group with unspecified viral infection and a third group with bacterial infection.

Results. In the analyzed period 72 children under 5 years of age who have shown signs of acute respiratory failure have been diagnosed with a specific viral infection, only $2 \%$ of the total viral infections. The main etiology was represented by respiratory syncytial virus (RSV) in $73.5 \%$ of cases, followed by Influenza $11 \%$, Parainfluenza 4.2\%, metapneumovirus (hMPV), Rhinovirus, Adenovirus and Bocavirus all with $2.8 \%$. Within the unspecified viral infection group antibiotics were used in $85.5 \%(n=53)$ of the cases. Within the specified viral infection group although viral etiology was firmly established antibiotics usage rate was higher $92 \%(n=57)$. Duration of hospital stay was 2,5 days higher in the viral specified group compared with the unspecified one. Thus we found that although the diagnosis of viral infection was certainly given because of severe clinical picture and young age of children the expenses were $30 \%$ higher.

Conclusions. In our study, RSV is the most common virus involved in the etiology of viral LRTI and is encountered in the most severe forms of the disease especially in young infants and prematures. Antibiotics are commonly used in the severe forms of LRTI, even if the viral etiology is certain and the groups of antibiotics used are similar. Determination of viral etiology has not reduced hospitalization, nor has led to changing of physician therapeutic approach.
\end{abstract}

Keywords: pnemonia, bronchiolitis, viral pneumonia, hospital cost, antibiotics

\section{INTRODUCTION}

Community-acquired pneumonia is one of the leading causes of death worldwide in children under 5 years. (1) WHO estimates that each year in the world there are 156 million cases of pneumonia in this age group, more than half of cases being reported in developing countries. (2) Of these, more than $50 \%$ of the cases require hospitalization, and $20 \%$ show severe forms of the disease. (3) Etiological diagnosis is still difficult to determine in recent years, however, the detection rate of viruses and pneumococcal infections in the lower respiratory tract (LRT) has improved considerably by using indirect immunofluorescence techniques, ELISA and PCR that are performed on nasopharyngeal aspira-

Corresponding author:

Alexandru-Ioan Ulmeanu, "Grigore Alexandrescu" Emergency Children's Hospital, 30-32 lancu de Hunedoara Blvd., Bucharest

E-mail: alex.ulmeanu@gmail.com 
tes of children. $(4,5)$ In practice, viral detection using multiplex PCR is becoming increasingly used, it is most useful to understand the epidemiology and impact of viruses on the pediatric population but there are still many debates regarding the influence of viral detection on patient care. (6)

\section{OBJECTIVES}

In this study we aimed to analyze the impact of viral etiology determination on the duration of hospitalization and on the administered treatment. As a secondary objective we wanted to compare severe LRTI of viral etiology with severe LRTI of bacterial etiology in children aged 0-5 years following anamnestic, clinical, laboratory parameteres and the administered treatment.

\section{MATERIAL AND METHODS}

We performed a retrospective study over a four year period, between April 2011 and April 2015 on patients admitted and treated in „Grigore Alexandrescu" Emergency Children Hospital, Bucharest. The selection criteria was: children between 0-5 years with severe lower respiratory tract infection. We selected only patients who had respiratory failure as secondary diagnosis. We have analyzed three groups of patients: a group with specified viral infection (children with the diagnosis of pneumonia and bronchiolitis in which viral assays were performed by RT-PCR and multiplex rapid tests for influenza or respiratory syncytial virus) a second group with unspecified viral infection (children who had at hospital discharge a diagnosis of pneumonia or bronchiolitis of unspecified viral etiology which was supported by clinical and laboratory criteria) and a third group with bacterial infection (children who had a diagnosis of bacterial infection which was supported by clinical and laboratory criteria). We excluded from the study: children with viral infection complicated by bacterial superinfection during hospitalization, children with neuromuscular diseases, immunodeficiency, congenital heart diseases, children with complicated bacterial infection, abscess, pleurisy, empyema, sepsis or meningitis. We analyzed charts, and data from the hospital statistical program. We analyzed data using Microsoft Office 2010 software package and SPSS v20. We compared the continuous variables with tstudent test and the categorical variables with odds ratio and Fischer's exact test, considering results with $p<0.05$ statistically significant.

\section{RESULTS AND DISCUSSION}

In the analyzed period there were 39,969 hospitalizations in the Pediatrics Clinic of SCUC „Grigore Alexandrescu" of them: 7,444 cases representing $18.6 \%$ were represented by lower respiratory tract infection. Viral etiology was diagnosed in 3,580 cases representing $8.9 \%$ of total. Bacterial etiology was diagnosed in 3,864 cases, representing $9.7 \%$ of total.

\section{Epidemiological data analysis}

In the analyzed period 72 children under 5 years of age who have shown signs of acute respiratory failure have been diagnosed with a specific viral infection, only $2 \%$ of the total viral infections. The main etiology was represented by respiratory syncytial virus (RSV) in $73.5 \%$ of cases, followed by Influenza $11 \%$, Parainfluenza $4.2 \%$, metapneumovirus (hMPV), Rhinovirus, Adenovirus and Bocavirus all with $2.8 \%$.

The average age of the studied group was 1 year and 2 months, $70 \%$ of the patients were infants and $7.5 \%$ of them newborns. Viruses are most commonly involved, at a rate of $30-67 \%$ in the etiology of LRTI in children 0-5 years, they are most common in infants where they produce severe bronchiolitis or pneumonias most commonly complicated with acute respiratory failure. (4)

We further compared the group of patients with specified viral infection with a group of patients (n $=72$ ) with bacterial pneumonia complicated by respiratory failure. The mean age was higher in bacterial group, 2 years and 1 month compared to 1 year and 2 months in viral group $(\mathrm{p}=0.0001)$, only $35 \%$ of cases were encountered in infants. (4) The etiology was determined in a proportion of $30 \%$ of the cases and it was represented by Streptoccocus pneumoniae $14 \%$, followed by Hemophilus influenzae $11 \%, 3 \%$ Stafiloccocus aureus and Klebsiella pneumoniae $1 \%$. The frequency of germs involved in LRTI of bacterial etiology was consistent with the literature reviews. In $70 \%$ of cases the etiology could not be determined. The etiology of severe LRTI encountered during the survey period is consistent with the literature reviews with the predominance of RSV infection and Streptoccocus pneumoniae in viral and bacterial LRTI of infants and children. (7-9).

We wanted to assess whether recurrent wheezing in the studied groups was more commonly associated with the viral infection and we found the following: recurrent wheezing in both groups (defined as more than 2 episodes of wheezing) was 
Clinical data analysis

\begin{tabular}{|l|c|c|c|c|c|c|}
\hline & $\begin{array}{c}\text { Nr. Cases } \\
\text { N = 144 }\end{array}$ & $\begin{array}{c}\text { OR Viral } \\
\text { Infection }\end{array}$ & $\begin{array}{c}\text { OR Bacterial } \\
\text { Infection }\end{array}$ & \multicolumn{2}{|c|}{ Confidence interval } & P value \\
\hline Recurrent wheezing & $64(45 \%)$ & 1.5 & - & 1.10 & 2.03 & $P<0.05$ \\
\hline Malnutrition & $38(26.4 \%)$ & 1.12 & - & 0.92 & 1.3 & $P>0.05$ \\
\hline Prematurity & $23(16 \%)$ & 1.87 & - & 0.8 & 4.1 & $P>0.05$ \\
\hline$<6$ months & $53(36.8 \%)$ & 1.45 & - & 1.12 & 1.89 & $P<0.05$ \\
\hline Dehydration & $71(49.3 \%)$ & - & 1.08 & 0.7 & 1.5 & $P>0.05$ \\
\hline Fever $>39 C$ & $53(36.8)$ & - & 1.45 & 1.12 & 1.89 & $P<0.05$ \\
\hline Leucocytosis & $67(46.5)$ & - & 2 & 1.38 & 3 & $P<0.05$ \\
\hline CRP & $69(47.9)$ & - & 2 & 1.36 & 2.92 & $P<0.05$ \\
\hline $\begin{array}{l}\text { Nasal carriage } \\
\text { Pneumococcus }\end{array}$ & $21(14 \%)$ & - & 3.2 & 1.23 & 8.27 & $P<0.05$ \\
\hline
\end{tabular}

present in 64 cases (44.4\%). Recurrent wheezing was present in the history of $40(55 \%)$ children with viral infection and only in $24(33 \%)$ of the cases with bacterial infection. In our study the risk of developing recurrent wheezing in children with viral infection was 1.5 times higher (95\% CI: 1.10 to 2.03) than in those with bacterial infection, result that was statistically significant. Literature studies correlate the presence of viral infection in infancy and particularly RSV infection with the risk of developing recurrent episodes of wheezing and asthma. There are still debates in the literature about causality connection asthma and viral infection and more longitudinal studies with more children involved are needed to accurately determine if viral infection in childhood is directly involved in the development of persistent asthma (10)

Fever is an important symptom used for distinguishing between bacterial infection and viral infection, so we further analyzed the presence of fever associated with respiratory symptoms and we found the following: the risk of having fever in a bacterial infection was not higher than viral infection, but fever with temperature over $39{ }^{\circ} \mathrm{C}$ was more frequent in the cases with bacterial infection $48 \%$ compared to $25 \%$ the risk of a having a temperature over $39{ }^{\circ} \mathrm{C}$ in bacterial infection was 1.45 times greater ( $95 \%$ CI 1.12 to 1.89 ), infants under 6 months presented with fever less often, the chance is 1,7 times higher for them not to develop fever (95\% CI 1.23 to 2.39). The european guidelines suggests that a corporeal temperature below $38^{\circ} 5 \mathrm{C}$ in a child under 5 years with symptoms suggestive of pneumonia having wheezing on auscultation should raise the suspicion of a viral infection. The results confirm the idea that a high fever suggests a bacterial infection but this should be carefully evaluated in young infants, were the absence of fever does not exclude a bacterial infection (11).

\section{Laboratory data analysis}

Leukocytosis with high neutrophil count was encountered more often in children with bacterial infection and together with an increased C-reactive protein it was positively correlated with bacterial infection, the chance of having elevated CRP was 2 times higher in bacterial infection (CI 1,36-2,92). The results are consistent with the results obtained in the literature, recent meta-analysis suggest that C-reactive protein may be used to distinguish between viral and bacterial pneumonia(12) C-reactive protein was increased in $23 \%$ of cases with viral infection, and of these especially in the children with RSV infection $24 \%(\mathrm{p}<0.05)$ and with influenza infection $62 \%(\mathrm{p}>0.05)$.

We have found that nasal pneumococcal colonization was found in $21(14 \%)$ cases, $22 \%(\mathrm{n}=16)$ with bacterial infections and $6 \%(n=5)$ with viral infection, the risk of having a bacterial infection was 3.2 times higher ( $95 \%$ CI 1.23 to 8.27 ). The confidence interval indicates a very significant result, it correlates with the debates that are found in literature reviews about nasal pneumococcal colonization. Although is not mandatory that the microbe discovered in the nasal swab is the germ involved in the etiology of pneumonia, colonization is actually the first stage of invasive pneumococcal infection and plays an essential role in the spread of bacteria in the community. (13)

\section{Analysis of treatment and hospitalization costs}

For the analysis of treatment and hospitalization costs we have added a third group for comparison which totaled 62 cases of children between $0-5$ years, with the average age of 2 years with a diagnosis of unspecified viral infection (pneumonia or bronchiolitis) complicated with acute respiratory failure. We compared this group with 62 children in the group with specified viral infection and with 62 
cases from the bacterial infection group. The results obtained were very interesting: within the unspecified viral infection group antibiotics were used in $85.5 \%(n=53)$ of the cases, the combination of at least two antibiotics was encountered in $3 \%$ of cases. The antibiotics used were aminopenicillins $35 \%$ of the cases, cephalosporins $48 \%$ with the 2 nd generation $24 \%$ and 3 rd generation $24 \%$, aminoglycosides $1.5 \%$ and macrolides $6 \%$ of the cases.

Within the specified viral infection group although viral etiology was firmly established antibiotics usage rate was higher $92 \%(n=57)$ this can be explained by the average age of the specified viral group, 1 year and 3 months compared to an average of 2 years, $70 \%$ infants with $48 \%$ less than 6 months compared to $30 \%$ infants with $10 \%$ less than 6 months. Aminopenicillins were used in a proportion of $53 \%$, cephalosporins $38 \%$, of which $30 \%-$ 3 rd generation and $8 \%$ - 2nd generation, aminoglycosides $-11 \%$, fluoroquinolones and carbapenems with $5 \%$. The combination of at least two antibiotics was found in $8 \%$ of the cases. The rate of antibiotic use is high in both groups and this can be explained by the severe forms of the disease and the young age of the children included in the study. Current recommendations suggest that antibiotics should not be used in viral infection unless a mixed infection is suspected, but some experts recommend using them in severe LRTI as secondary bacterial infection can sometimes be difficult to rule out. A major randomized study double-blind placebo-controlled has shown, however, that antibiotics used during the RSV infection did not alter the course of the disease or prevent the development of complications (5)

In the group with bacterial infection, antibiotics were used in all cases $(n=62)$. The combination of the antibiotic has been used for more than $70 \%$ of cases $(p<0.05)$. The need of using a combination of antibiotics in the bacterial infection was 2.9 fold greater than in the viral infection (95\% CI 1.98 to 4.25).

Duration of hospital stay averaged $6.69 \pm 3.6$ in the group with specified viral infection, with a minimum of 2 days and a maximum of 23 days. In the group with unspecified viral infection the average length of stay was $4 \pm 1.74$ with 10 days maximum and 1 day minimum stay. The average length of stay for the bacterial infection group was $9.65 \pm 3.3$ minimum 5 days up to 20 days. ( $<<0.0001)$. Although viral etiology was firmly established hospitalization was longer with two and a half day, this can be explained by the high number of young aged children with severe forms of the disease, a longer prospective study is needed to assess the exact influence of viral etiology determination on hospital stay.

Hospitalization expenses were $1166 \pm 461 \mathrm{RON}$ for the unspecified viral infection group, $1542 \pm$ 522 RON for the group with viral determination and $3148 \pm 1226 \mathrm{RON}$ for the group with bacterial infection. Thus we found that although the diagnosis of viral infection was certainly given severe clinical picture and young age of children the expenses were $30 \%$ higher $(p<0.0002)$. For the bacterial infection group costs were almost double $3148 \pm 1226$ RON. Studies show conflicting data in the literature related to the efficiency of viral determinations in practice. In some studies the rapid antigen tests were effective in reducing the rate of hospitalizations and the rate of antibiotic prescribing. A study conducted into the ER did not confirm the results, showing that diagnostic tests must be used in specific departments to provide most benefits. $(14,15)$. Latest controlled studies show that although many types of viruses are diagnosed with RT-PCR multiplex, this tool does not reduce the number of admissions, length of stay, and does not limit the use of antibiotics. $(16,17)$. Current guidelines strongly recommend the use of viral determination tests for evidence of influenza virus, but in practice multiple viral determination by RT-PCR should only be used if the clinician believes that the result will change his therapeutic approach (18).

\section{CONCLUSIONS}

Although severe respiratory infections of viral etiology represent a significant percentage of all hospitalizations performed in small children, the etiological diagnosis is rarely established, because modern methods of diagnosis like RT-PCR are not in the reach of the medical practitioner. In our study, RSV is the most common virus involved in the etiology of viral LRTI and is encountered in the most severe forms of the disease especially in young infants and prematures. Antibiotics are commonly used in the severe forms of LRTI, even if the viral etiology is certain and the groups of antibiotics used are similar. Determination of viral etiology has not reduced hospitalization, nor has led to changing of physician therapeutic approach. The costs of hospitalization were higher in those with specified viral infection but were substantially lower than those found in LRTI of bacterial etiology. Although the utility in the clinical management of the sick child remains to be established, the deter- 
mination of viral etiology by RT-PCR remains an important tool for better understanding of the etiology of lower respiratory tract infections of the child and proves to have an important role in surveillence of epidemics and prevention of hospital infections.

\section{REFERENCES}

1. J. Bryce, C. Boschi-Pinto, K. Shibuya, R.E. Black, WHO estimates of the causes of death in children, Lancet, 365 (2005), pp. 1147-1152

2. Rudan I., Boschi-Pinto C, Biloglav Z, et al. Epidemiology and etiology of childhood pneumonia. Bulletin of World Health Organisation 2008; 86:408.

3. Barson W. Pneumonia in children: Epidemiology, pathogenesis, and etiology in UptoDate accessed May 2015

4. Manikam L., Lakhanpaul M., Epidemiology of community acquired pneumonia, Paediatrics and Child Health, Vol 22, Issue 7, July 2012, p. $299-306$

5. Ruuskanen O., Lahti E., Jennings L., Murdoch D. Viral pneumonia, Lancet 2011; 377: 1264-75

6. Emilie Vallières, Christian Renaud, Clinical and economical impact of multiplex respiratory virus assays, Diagnostic Microbiology and Infectious Disease, Vol 76, Issue 3, July 2013, p 255-261

7. P. Pattemore, Lance Jennings, Epidemiology of Respiratory Infections in Pediatric Respiratory Medicine, eds Taussig, Landau, 2nd edition, Mosby- Elsevier, 2011, pg. 435-452

8. Boyer K.M. Nonbacterial pneumonia, Textbook of Pediatric Infectious Diseases, 6th, Feigin RD, Cherry J.D., Demmler H., Kaplan S.L., Saunders, 2009. p.289

9. Harris M., Clark J., Coote N., Fletcher P., British Thoracic Society guidelines for the management of community acquired pneumonia in children: update 2011, Thorax. 2011;66 Suppl 2

10. Kecia N. Carroll, Tina V. Hartert, The Impact of Respiratory Viral Infection on Wheezing IIInesses and Asthma Exacerbations, Immunol Allergy Clin North Am. 2008 Aug; 28(3): 539-561.

11. British Thoracic Society of Standards of Care Committee, BTS guidelines for the management of community acquired pneumonia in childhood, Thorax, 57 (suppl 1) (2002), pp. i1-24

\section{Acknowledgment}

This paper is supported by SOP HRD, financed from the European Social Fund and by the Romanian Government under the contract number POSDRU/159/1.5/S/137390

12. Flood R., Badik J., Aronoff S. The utility of serum C-reactive protein in differentiating bacterial from nonbacterial pneumonia in children, Pediatric Infectious Disease Journal, 27 (2008), pp. 95-99

13. Bogaert D., De Groot R., Hermans P.W. Streptococcus pneumoniae colonisation: the key to pneumococcal disease, Lancet Infectious Diseases. 2004 Mar; 4(3):144-54.

14. Q. Doan, P. Enarson, N. Kissoon, T.P. Klassen, D.W. Johnson, Rapid viral diagnosis for acute febrile respiratory illness in children in the Emergency Department, Cochrane Database Syst Rev, 5 (2012)

15. Vallières E., Renaud C. Clinical and economical impact of multiplex respiratory virus assays, Diagn Microbiol Infect Dis. 2013 Jul; 76(3):255-61.

16. J. Oosterheert, A.M. van Loon, R. Schuurman, A.I. Hoepelman, E. Hak, S. Thijsen, et al., Impact of rapid detection of viral and atypical bacterial pathogens by real-time polymerase chain reaction for patients with lower respiratory tract infection, Clin Infect Dis, 41 (10) (2005), p. 1438-1444

17. J.O. Wishaupt, A. Russcher, L.C. Smeets, F.G. Versteegh, N.G. Hartwig, Clinical impact of RT-PCR for pediatric acute respiratory infections: a controlled clinical trial, Pediatrics, 128 (5) (2011), p. e1113-e1120

18. John S.. Bradley, Carrie L. Byington, Samir S. Shah et al. The Management of Community-Acquired Pneumonia in Infants and Children Older Than 3 Months of Age: Clinical Practice Guidelines by the PIDS and the IDSA, IDSA Practice Guidelines 